\title{
Intuitive Approximations in Discrete Renewal Theory Part 1: regularly varying case
}

\author{
Edward Omey \& Stefan Van Gulck* \\ KU Leuven, Faculty of Economics and Business - Campus Brussels \\ Warmoesberg 26, 1000 Brussels, Belgium
}

\begin{abstract}
It is usually impossible to find explicit expressions for the renewal sequence. This paper presents a simple method to approximate the renewal sequence, which covers many of the known approximations. The paper uses the ideas of Mitov and Omey (2014).
\end{abstract}

Keywords: renewal sequence, regular variation, approximations 2000 MSC: 60K05, 60E10, 26A12

\section{Introduction}

Suppose that $X, X_{1}, X_{2}, \ldots$ are i.i.d. nonnegative integer-valued random variables with p.d.f. $p_{k}=P(X=k), k \in \mathbb{N}_{0}$. The d.f. of $X$ is given by $F(x)=P(X \leq x)$ and its tail is denoted by $\bar{F}(x)=1-F(x)$. Throughout the paper we assume that $\left(p_{k}\right)$ is aperiodic: $\operatorname{gcd}\left\{k: p_{k}>0\right\}=1$. We also assume that $0<\mu=E(X)<\infty$. For $n \in \mathbb{N}_{0}$, the partial sums $S_{n}$ are given by $S_{0}=0$ and $S_{n}=X_{1}+\cdots+X_{n}$ for $n \geq 1$. Note that $P\left(S_{n} \leq x\right)=F^{\star n}(x)$, which is the $n$-fold convolution of $F$, i.e. $F^{\star 0}=1_{[0, \infty)}$ and $F^{\star n}=F \star F^{\star(n-1)}$, where the convolution of two d.f. is defined by $F \star G(x)=\int_{0}^{x} F(x-y) d G(y)$. For briefness, we write $F^{\star n}(x)$ as $F_{k}^{\star n}$ in case $x$ is a nonnegative integer $k$. Moreover, $P\left(S_{n}=k\right)=p_{k}^{* n}$, which is the $n$-fold convolution of $\left(p_{k}\right)$, i.e. $p_{k}^{* 0}=1_{\{0\}}(k)$ and $p_{k}^{* n}=\left(p * p^{*(n-1)}\right)_{k}$, where the convolution of two sequences $\left(a_{k}\right)$ and $\left(b_{k}\right)$ is defined by $(a * b)_{k}=\sum_{i=0}^{k} a_{i} b_{k-i}$. The generating function of $X$ is $\hat{P}(z)=E\left(z^{X}\right),|z|<1$, and $\hat{P}(1)=1$. The generating function of $S_{n}$ is given by $\hat{P}^{n}(z)$. Since $X$ has finite expectation, we have $\mu=\hat{P}^{\prime}(1)$.

\footnotetext{
*Corresponding author: stefan.vangulck@kuleuven.be
} 
Let $X_{e}$ be a random variable, independent of $X$, that has the equilibrium distribution corresponding to $X$, i.e. $p_{e, k}=P\left(X_{e}=k\right)=\bar{F}_{k} / \mu$ for $k \in \mathbb{N}_{0}$. The generating function of $X_{e}$ satisfies $\hat{P}_{e}(z)=(1-\hat{P}(z)) /(\mu(1-z))$. Define $S_{e, n}=X_{e, 1}+\cdots+X_{e, n}, p_{e, k}^{* n}$, and $F_{e, k}^{\star n}$ analogously as above.

The renewal sequence $\left(u_{n}\right)$ is defined by $u_{n}=\sum_{k=0}^{\infty} p_{n}^{* k}$. The aim of the present paper is to obtain approximations for $u_{n}$ when $n$ is large. Therefore, all limits that appear later are taken with respect to $n \rightarrow \infty$. It is well known that $u_{n} \rightarrow 1 / \mu$ and the main problem is to obtain precise estimates for the rate at which $u_{n}-1 / \mu \rightarrow 0$ or $\Delta u_{n}=u_{n-1}-u_{n} \rightarrow 0$.

In our approach, we start from the generating function of $\left(u_{n}\right)$, which is given by $\hat{U}(z)=\sum_{n=0}^{\infty} u_{n} z^{n}=(1-\hat{P}(z))^{-1}=\left(\mu(1-z)\left(1-\left(1-\hat{P}_{e}(z)\right)\right)\right)^{-1}$. Using a Taylor expansion, we obtain that

$$
\hat{U}(z)=\sum_{k=0}^{\infty} \hat{T}_{k}(z), \quad \text { with } \quad \hat{T}_{k}(z)=\frac{1}{\mu(1-z)}\left(1-\hat{P}_{e}(z)\right)^{k} .
$$

Formula (1) suggests the following approximations $\hat{U}_{m}(z)$ for $\hat{U}(z): \hat{U}_{m}(z)=$ $\sum_{k=0}^{m} \hat{T}_{k}(z)$. By inversion, this approach then leads to approximations $u_{m, n}$ for the renewal sequence $u_{n}$ of the form $u_{m, n}=\sum_{k=0}^{m} t_{k, n}$, where the sequence $\left(t_{k, n}\right)$ has generating function $\hat{T}_{k}(z)=\sum_{n=0}^{\infty} t_{k, n} z^{n}$. In the next section we will identify $\hat{T}_{k}(z)$ and $\left(t_{k, n}\right)$. In this paper we focus on the cases $0 \leq m \leq 3$ and show that our approximations $\left(u_{m, n}\right)$ correspond to the approximations that have been published in many papers before.

\section{The sequences $\left(t_{k, n}\right)$ and $\left(\delta_{k, n}\right)$}

\subsection{Expressions for $\left(t_{k, n}\right)$ and $\left(\delta_{k, n}\right)$}

We first identify $\hat{T}_{k}(z)$. If $k=0$, then (1) gives $\hat{T}_{0}(z)=1 /(\mu(1-z))$, which shows that $\hat{T}_{0}(z)$ is the generating function of $t_{0, n}=1 / \mu$. For $k \geq 1$, the binomial expansion in (1) yields

$$
\begin{aligned}
\hat{T}_{k}(z) & =\frac{1}{\mu(1-z)} \sum_{i=0}^{k}\left(\begin{array}{c}
k \\
i
\end{array}\right)(-1)^{i} \hat{P}_{e}^{i}(z) \\
& =\frac{-1}{\mu(1-z)} \sum_{i=1}^{k}\left(\begin{array}{c}
k \\
i
\end{array}\right)(-1)^{i}\left(1-\hat{P}_{e}^{i}(z)\right) .
\end{aligned}
$$

Since $\hat{P}_{e}^{i}(z)$ is the generating function of $S_{e, i}$, we have $\left(1-\hat{P}_{e}^{i}(z)\right) /(1-z)=$ $\sum_{n=0}^{\infty} \frac{e}{F_{e, n}^{\star i}} z^{n}$. We therefore obtain the following result. 
Lemma 1. For $n \geq 1$, let $\delta_{k, n}=t_{k, n-1}-t_{k, n}$. Then $t_{0, n}=1 / \mu, \delta_{0, n}=0$ and, if $k \geq 1$,

$$
\begin{aligned}
t_{k, n} & =-\frac{1}{\mu} \sum_{i=1}^{k}\left(\begin{array}{c}
k \\
i
\end{array}\right)(-1)^{i} \overline{F_{e, n}^{\star i}}, \\
\delta_{k, n} & =-\frac{1}{\mu} \sum_{i=1}^{k}\left(\begin{array}{c}
k \\
i
\end{array}\right)(-1)^{i} p_{e, n}^{* i} .
\end{aligned}
$$

We now consider into detail the cases $k=1,2,3$.

Lemma 2. For $k \geq 2$, let $R_{k, n}^{e}=\overline{F_{e, n}^{\star k}}-k \bar{F}_{e, n}$ and $r_{k, n}^{e}=p_{e, n}^{* k}-k p_{e, n}$. Then

$$
\begin{array}{ll}
t_{1, n}=\bar{F}_{e, n} / \mu & \delta_{1, n}=p_{e, n} / \mu \\
t_{2, n}=-R_{2, n}^{e} / \mu & \delta_{2, n}=-r_{2, n}^{e} / \mu \\
t_{3, n}=\left(R_{3, n}^{e}-3 R_{2, n}^{e}\right) / \mu & \delta_{3, n}=\left(r_{3, n}^{e}-3 r_{2, n}^{e}\right) / \mu
\end{array}
$$

Proof. The results follow directly from (2) and (3).

\subsection{Asymptotic behaviour of $\left(t_{k, n}\right)$ and $\left(\delta_{k, n}\right), 1 \leq k \leq 3$.}

In order to discuss the asymptotic behaviour of $\left(t_{k, n}\right)$ and $\left(\delta_{k, n}\right)$, we recall some basic definitions and properties of regularly varying sequences.

\subsubsection{Regularly varying sequences}

A sequence of real numbers $\left(a_{n}\right)$ is regularly varying at infinity and with real index $\alpha$ if $a_{n}>0$ for $n$ large and if

$$
\lim _{x \rightarrow \infty} \frac{a_{[x y]}}{a_{[x]}}=y^{\alpha}, \quad \forall y>0 .
$$

Notation: $\left(a_{n}\right) \in R S(\alpha)$. We write $\left(a_{n}\right) \in R S$ if $\left(a_{n}\right) \in R S(\alpha)$ for some $\alpha \in \mathbb{R}$. If $\left(a_{n}\right) \in R S(\alpha)$, then (4) holds locally uniformly in $y>0$; see sections 1.2 and 1.9 in Bingham et al. (1987). From this it follows that $R S \subset L S$, where a sequence of real numbers $\left(a_{n}\right)$ is in the class $L S$ if $a_{n}>0$ for $n$ large and if $a_{n+1} / a_{n} \rightarrow 1$. If $\left(a_{n}\right) \in R S(\alpha)$, then for each $\epsilon>0$ we can find constants $A, B, x^{\circ}$ so that

$$
A y^{\alpha-\epsilon} \leq \frac{a_{[x y]}}{a_{[x]}} \leq B y^{\alpha+\epsilon}, \quad \forall x \geq x^{\circ}, \forall y \geq 1,
$$

cf. section 1.5 in Bingham et al. (1987) or Proposition 1.7 in Geluk and de Haan (1987). For sequences $\left(a_{n}\right)$ and $\left(b_{n}\right)$, we use the following notations: 
- $a_{n}=O(1) b_{n}$ means that $\lim \sup _{n \rightarrow \infty} a_{n} / b_{n}<\infty$;

- $a_{n}=o(1) b_{n}$ means that $\lim \sup _{n \rightarrow \infty} a_{n} / b_{n}=0$;

- $a_{n} \approx b_{n}$ means that $a_{n}=O(1) b_{n}$ and $b_{n}=O(1) a_{n}$;

- $a_{n} \sim b_{n}$ means that $\lim _{n \rightarrow \infty} a_{n} / b_{n}=1$.

Note that for $\left(a_{n}\right) \in R S(\alpha)$ we have $a_{n}=o(1) n^{\alpha+\epsilon}$. If $\alpha+\beta<-1$, then Karamata's theorem states that $\sum_{n=0}^{\infty} n^{\beta} a_{n}<\infty$ and

$$
\sum_{k=n}^{\infty} k^{\beta} a_{k} \sim-\frac{n^{\beta+1} a_{n}}{\alpha+\beta+1},
$$

cf. section 1.6 in Bingham et al. (1987). Using local uniform convergence, it also follows that for $B \geq A>0$ we have

$$
\sum_{k=[A n]}^{[B n]} k^{\beta} a_{k} \approx n^{\beta+1} a_{n} .
$$

The next lemma will be used in the sections below.

Lemma 3. Suppose that $\left(a_{n}\right) \in R S(-\alpha)$ and $\left(b_{n}\right) \in R S$.

(i) If $\alpha>1$, then $\sum_{k=0}^{[n / 2]} b_{n-k} a_{k} / b_{n} \rightarrow \sum_{k=0}^{\infty} a_{k}$;

(ii) If $\alpha>2$, then $\sum_{k=0}^{[n / 2]} \sum_{i=0}^{k} b_{n-i} a_{k} / b_{n} \rightarrow \sum_{k=0}^{\infty} k a_{k}$.

Proof. Note that $\alpha>1$ implies that $\sum_{k=0}^{\infty} a_{k}<\infty$ and that $\alpha>2$ implies that $\sum_{k=0}^{\infty} k a_{k}<\infty$.

(i) Because $\left(b_{n}\right) \in L S$, we have $b_{n-k} / b_{n} \rightarrow 1$ for all $k$. Since $0 \leq k \leq[n / 2]$, $[n / 2] \leq n-[n / 2] \leq n-k \leq n$. From $\left(b_{n}\right) \in R S$ now follows that

$$
\frac{b_{n-k}}{b_{n}} \leq \sup _{1 / 2 \leq x \leq 1} \frac{b_{[n x]}}{b_{n}} \leq C, \quad n \geq n^{\circ} .
$$

We can use Lebesgue's theorem to conclude that

$$
\frac{1}{b_{n}} \sum_{k=0}^{[n / 2]} b_{n-k} a_{k} \rightarrow \sum_{k=0}^{\infty} a_{k} .
$$


(ii) As in (i) we have $b_{n-i} / b_{n} \rightarrow 1$, for all $i$, and for each fixed $k$ we have $\sum_{i=0}^{k} b_{n-i} / b_{n} \rightarrow k$. Now we have

$$
\frac{1}{b_{n}} \sum_{i=0}^{k} b_{n-i} \leq \sup _{n-k \leq j \leq n} \frac{b_{j}}{b_{n}} k \leq C k, \quad n \geq n^{\circ}
$$

We can again use Lebesgue's theorem to obtain result (ii).

\subsubsection{Asymptotic behaviour of $\left(t_{1, n}\right)$ and $\left(\delta_{1, n}\right)$}

Recall from Lemma 2 that $t_{1, n}=\bar{F}_{e, n} / \mu$ and $\delta_{1, n}=p_{e, n} / \mu=\bar{F}_{n} / \mu^{2}$. We now apply Karamata's theorem, cf. (5), to obtain the following result.

Lemma 4. (i) If $\left(\bar{F}_{n}\right) \in R S(-\alpha)$ and $\alpha>1$, then $\mu<\infty, \delta_{1, n}=\bar{F}_{n} / \mu^{2}$, and $t_{1, n} \sim n \bar{F}_{n} /\left(\mu^{2}(\alpha-1)\right)$.

(ii) If $\left(p_{n}\right) \in R S(-\alpha)$ and $\alpha>2$, then $\mu<\infty, \bar{F}_{n} \sim n p_{n} /(\alpha-1), \delta_{1, n} \sim$ $n p_{n} /\left(\mu^{2}(\alpha-1)\right)$, and $t_{1, n} \sim n^{2} p_{n} /\left(\mu^{2}(\alpha-1)(\alpha-2)\right)$.

Remark 1. From $t_{1, n}=\bar{F}_{e, n} / \mu$ and $\delta_{1, n}=\bar{F}_{n} / \mu^{2}$ (cf. Lemma 2), we also obtain that

$$
\begin{aligned}
& \sum_{n=1}^{\infty} n^{r} t_{1, n}=\frac{1}{\mu} \sum_{n=1}^{\infty} n^{r} \bar{F}_{e, n} \leq C E\left(X_{e}^{r+1}\right), \\
& \sum_{n=1}^{\infty} n^{r} \delta_{1, n}=\frac{1}{\mu^{2}} \sum_{n=1}^{\infty} n^{r} \bar{F}_{n} \leq C E\left(X^{r+1}\right) .
\end{aligned}
$$

\subsubsection{Asymptotic behaviour of $\left(t_{2, n}\right)$ and $\left(\delta_{2, n}\right)$}

Recall from Lemma 2 that $t_{2, n}=-R_{2, n}^{e} / \mu$ and $\delta_{2, n}=-r_{2, n}^{e} / \mu$, where $R_{2, n}^{e}=$ $P\left(X_{e, 1}+X_{e, 2}>n\right)-2 P\left(X_{e}>n\right)$ and $r_{2, k}^{e}=P\left(X_{e, 1}+X_{e, 2}=n\right)-2 P\left(X_{e}=n\right)$, with $X_{e, 1}$ and $X_{e, 2}$ i.i.d. copies of $X_{e}$.

Two lemmas. In the first result we study more generally the asymptotic behaviour of $R_{2, n}$ and $r_{2, n}$, where

$$
\begin{aligned}
R_{2, n} & =P(X+Y>n)-P(X>n)-P(Y>n), \\
r_{2, n} & =P(X+Y=n)-P(X=n)-P(Y=n),
\end{aligned}
$$

and where $X$ and $Y$ are independent discrete r.v. with $a_{n}=P(X=n)$ and $b_{n}=P(Y=n), n \geq 0$. For further use, starting from $\left(a_{n}\right)$, we define 
$\delta_{n}^{a}=a_{n-1}-a_{n}, n \geq 1$. Clearly, for $j>i$, we have

$$
\sum_{n=i+1}^{j} \delta_{n}^{a}=a_{i}-a_{j} \quad \text { and } \quad \sum_{n=i+1}^{\infty} \delta_{n}^{a}=a_{i} .
$$

We use a similar notation for $\left(b_{n}\right)$. Note that $R_{2, n}=\sum_{k=n+1}^{\infty} r_{2, k}$ and that $P(X+Y=n)=(a * b)_{n}$. First we consider $R_{2, n}$ and $(a * b)_{n}$.

Lemma 5. Assume that $\left(a_{n}\right) \in R S(-\alpha)$ and $\left(b_{n}\right) \in R S(-\beta)$, with $\alpha>2$ and $\beta>2$.

(i) We have $E(X)+E(Y)<\infty$ and

$$
R_{2, n}=b_{n} E(X)+a_{n} E(Y)+o(1) a_{n}+o(1) b_{n} ;
$$

(ii) $(a * b)_{n}=a_{n}+b_{n}+o(1) a_{n}+o(1) b_{n}$.

Proof. Let $m=[n / 2]$.

(i) We have $R_{2, n}=I+I I+I I I-I V$, where

$$
\begin{aligned}
I & =P(X+Y>n, m<Y \leq n, X \leq m) \\
I I & =P(X+Y>n, m<X \leq n, Y \leq m) \\
I I I & =P(m<X \leq n, m<Y \leq n) \\
I V & =P(X>n, Y>n) .
\end{aligned}
$$

First we consider $I$. We clearly have

$$
I=\sum_{k=0}^{m} P(n-k<Y \leq n) P(X=k)=\sum_{k=0}^{m} \sum_{i=0}^{k-1} b_{n-i} a_{k} .
$$

Using Lemma 3 (ii) we obtain that $I / b_{n} \rightarrow \sum_{k=0}^{\infty} k a_{k}=E(X)$. In a similar way, we obtain that $I I / a_{n} \rightarrow E(Y)$. Now consider III. Since $m=[n / 2]$ and $\left(a_{n}\right),\left(b_{n}\right) \in R S$, we have, cf. (6),

$$
I I I=\sum_{i=m+1}^{n} a_{i} \sum_{j=m+1}^{n} b_{j} \approx n^{2} a_{n} b_{n} .
$$

Since $\alpha>2$, it follows that $n^{2} a_{n} \rightarrow 0$ and $I I I=o(1) b_{n}$. Finally, for $I V$ we have $P(X>n) \approx n a_{n}$ and $P(Y>n) \approx n b_{n}$, cf. (5), and it therefore follows that $I V \approx n^{2} a_{n} b_{n}=o(1) a_{n}$. Combining the estimates for $I, I I$, $I I I$, and $I V$, result (i) follows. 
(ii) We have

$$
(a * b)_{n}=\sum_{k=0}^{m} a_{k} b_{n-k}+\sum_{k=0}^{n-m-1} a_{n-k} b_{k}=I+I I .
$$

Using Lemma 3 (i) we have $I / b_{n} \rightarrow \sum_{k=0}^{\infty} a_{k}=1$ and $I I / a_{n} \rightarrow 1$.

In our next result we discuss $r_{2, n}$.

Lemma 6. Suppose that $\left(\delta_{n}^{a}\right) \in R S(-\alpha)$ and $\left(\delta_{n}^{b}\right) \in R S(-\beta)$, with $\alpha>3$ and $\beta>3$. Then $r_{2, n}=\delta_{n}^{b} E(X)+\delta_{n}^{a} E(Y)+o\left(\delta_{n}^{a}\right)+o\left(\delta_{n}^{b}\right)$.

Proof. Using $m=[n / 2]$, we have $r_{2, n}=I+I I$, where

$$
I=\sum_{k=0}^{m} a_{k} b_{n-k}-b_{n} \quad \text { and } \quad I I=\sum_{k=0}^{n-m-1} a_{n-k} b_{k}-a_{n} .
$$

First consider $I$. We have

$$
I=\sum_{k=1}^{m} a_{k}\left(b_{n-k}-b_{n}\right)-b_{n} \sum_{k=m+1}^{\infty} a_{k}=I_{A}-I_{B} .
$$

Using $b_{n-k}-b_{n}=\sum_{i=0}^{k-1} \delta_{n-i}^{b}$, we have $I_{A}=\sum_{k=1}^{m} a_{k} \sum_{i=0}^{k-1} \delta_{n-i}^{b}$. Lemma 3 (ii) shows that $I_{A} / \delta_{n}^{b} \rightarrow E(X)$. Now consider $I_{B}$. Using $\sum_{k=n+1}^{\infty} \delta_{k}^{b}=b_{n}$, $\left(\delta_{n}^{b}\right) \in R S(-\beta)$ with $\beta>3$, we have $b_{n} \approx n \delta_{n}^{b}$ and $\left(b_{n}\right) \in R S(1-\alpha)$. Similarly, $a_{n} \approx n \delta_{n}^{a}$ and $\left(a_{n}\right) \in R S(1-\alpha)$. Since $\alpha-1>2$, it follows that $\sum_{k=n+1}^{\infty} a_{k} \approx n a_{n} \approx n^{2} \delta_{n}^{a}$. Combining these estimates, it follows that

$$
I_{B} \approx n \delta_{n}^{b} \sum_{k=m+1}^{\infty} a_{k} \approx n \delta_{n}^{b} m^{2} \delta_{m}^{a}
$$

Since $m=[n / 2],\left(\delta_{n}^{a}\right) \in R S$, and $\beta>3$, we conclude that $I_{B} \approx n^{3} \delta_{n}^{b} \delta_{n}^{a}=$ $o(1) \delta_{n}^{a}$. We similarly find that $I I / \delta_{n}^{a} \rightarrow E(Y)$.

Asymptotic behaviour of $\left(t_{2, n}\right)$ and $\left(\delta_{2, n}\right)$. We apply Lemma 5 and Lemma 6 , with $a_{n}=b_{n}=p_{e, n}$ and $\delta_{n}^{a}=p_{e, n-1}-p_{e, n}=\left(\bar{F}_{n-1}-\bar{F}_{n}\right) / \mu=p_{n} / \mu$.

Theorem 7. (i) If $\left(p_{e, n}\right) \in R S(-\alpha)$, with $\alpha>2$, then $t_{2, n} \sim-2 \mu_{e} p_{e, n} / \mu$.

(ii) If $\left(p_{n}\right) \in R S(-\alpha)$, with $\alpha>3$, then $p_{e, n} \sim n p_{n} /(\mu(\alpha-1)), \delta_{2, n} \sim$ $-2 \mu_{e} p_{n} / \mu^{2}$, and $t_{2, n} \sim-2 n p_{n} \mu_{e} /\left(\mu^{2}(\alpha-1)\right)$. 
Proof. Recall from Lemma 2 that $t_{2, n}=-R_{2, n}^{e} / \mu$ and $\delta_{2, n}=-r_{2, n}^{e} / \mu$.

(i) Lemma 5 (i) shows that $R_{2, n}^{e} \sim 2 \mu_{e} p_{e, n}$.

(ii) $\left(p_{n}\right) \in R S(-\alpha), \alpha>3$, and (5) yield $p_{e, n}=\bar{F}_{n} / \mu \sim n p_{n} /(\mu(\alpha-1))$. This also shows that $\left(p_{e, n}\right) \in R S(-(\alpha-1))$. The result for $t_{2, n}$ thus follows from (i). Since $\left(\delta_{n}^{a}\right)=\left(p_{n} / \mu\right) \in R S(-\alpha)$ and $\alpha>3$, Lemma 6 shows that $\delta_{2, n}=-r_{2, n}^{e} / \mu \sim-2 \delta_{n}^{a} \mu_{e} / \mu=-2 p_{n} \mu_{e} / \mu^{2}$.

Remark 2. Note that

$$
\sum_{n=1}^{\infty} n^{r}\left|t_{2, n}\right| \leq C \sum_{n=1}^{\infty} n^{r}\left|R_{2, n}^{e}\right| \leq C\left(E\left(X_{e}+Y_{e}\right)^{r+1}+E X_{e}^{r+1}\right) .
$$

\subsubsection{Asymptotic behaviour of $\left(t_{3, n}\right)$}

Recall from Lemma 2 that $t_{3, n}=\left(R_{3, n}^{e}-3 R_{2, n}^{e}\right) / \mu$.

One lemma. We first discuss, more generally, the asymptotic behaviour of $A_{n}=R_{3, n}-3 R_{2, n}$, where $R_{2, n}=P(X+Y>n)-2 P(X>n), R_{3, n}=P(X+$ $Y+Z>n)-3 P(X>n)$, and $X, Y$ and $Z$ are i.i.d. with $a_{n}=P(X=n)$. We now use $\delta_{n}=a_{n-1}-a_{n}$.

Lemma 8. If $\left(\delta_{n}\right) \in R S(-\alpha)$ with $\alpha>3$, then $A_{n} / \delta_{n} \rightarrow 3(E(X))^{2}$.

Proof. Direct calculations show that

$$
A_{n}=\sum_{k=0}^{n} R_{2, n-k} a_{k}-R_{2, n}=I+I I
$$

where (using $m=[n / 2]$ )

$$
I=\sum_{k=0}^{m} R_{2, n-k} a_{k}-R_{2, n} \quad \text { and } \quad I I=\sum_{k=m+1}^{n} R_{2, n-k} a_{k} .
$$

Clearly,

$$
I=\sum_{k=0}^{m}\left(R_{2, n-k}-R_{2, n}\right) a_{k}-R_{2, n} \sum_{k=m+1}^{\infty} a_{k}=I_{A}-I_{B}
$$

and

$$
I I=\sum_{k=0}^{n-m-1} R_{2, k}\left(a_{n-k}-a_{n}\right)+a_{n} \sum_{k=0}^{n-m-1} R_{2, k}=I I_{A}+I I_{B} .
$$


First consider $I_{A}$. We have $R_{2, n-k}-R_{2, n}=\sum_{j=0}^{k-1} r_{2, n-j}$. Lemma 6 yields $r_{2, n} \sim 2 \delta_{n} E(X) \in R S(-\alpha)$. Lemma 3 (ii) shows that $I_{A} / \delta_{n} \rightarrow 2(E(X))^{2}$. Next consider $I_{B}$. Lemma 5 (i) shows that $R_{2, n} \approx a_{n}$. Since $\left(a_{n}\right) \in R V(1-\alpha)$, it follows that $I_{B} \approx a_{n} \sum_{k=m}^{\infty} a_{k} \approx n a_{n}^{2}$. Since $a_{n} \approx n \delta_{n}$ and $\alpha>3$, we obtain that $I_{B}=o\left(\delta_{n}\right)$.

Now consider $I I_{A}$. Using $a_{n-k}-a_{n}=\sum_{i=0}^{k-1} \delta_{n-i}$ and $\left(\delta_{n}\right) \in R S(-\alpha) \subset$ $L S$, Lemma 3 (ii) gives $I I_{A} / \delta_{n} \rightarrow \sum_{k=0}^{\infty} k R_{2, k}$. Remark 3 below shows that $\sum_{k=0}^{\infty} k R_{2, k}=(E(X))^{2}$. Finally consider $I I_{B}$. Since $\sum_{n=0}^{\infty} R_{2, n}=0$, we have $I I_{B}=-a_{n} \sum_{k=n-m}^{\infty} R_{2, k}$. Using $R_{2, n} \approx a_{n}$, cf. Lemma 5, and $\left(a_{n}\right) \in$ $R V(1-\alpha)$ with $\alpha>3$, we have $I I_{B} / \delta_{n} \approx n a_{n}^{2} / \delta_{n} \approx n^{3} \delta_{n} \rightarrow 0$.

Remark 3. For the sequence $\left(R_{2, n}\right)$ we have

$$
\hat{R}_{2}(z)=\sum_{n=0}^{\infty} z^{k} R_{2, k}=-\frac{(1-\hat{P}(z))^{2}}{1-z} .
$$

If $\mu<\infty$, we have

$$
\lim _{z \rightarrow 1} \hat{R}_{2}(z)=-\lim _{z \rightarrow 1} \frac{1-\hat{P}(z)}{1-z}(1-\hat{P}(z))=-\mu \times 0=0,
$$

or $\hat{R}_{2}(1)=0$. Using this result, we get

$$
\hat{R}_{2}^{\prime}(1)=\lim _{z \rightarrow 1} \frac{\hat{R}_{2}(1)-\hat{R}_{2}(z)}{1-z}=\lim _{z \rightarrow 1} \frac{(1-\hat{P}(z))^{2}}{(1-z)^{2}}=\mu^{2} .
$$

Asymptotic behaviour of $\left(t_{3, n}\right)$. Applying Lemma 8 gives the following result.

Theorem 9. If $\left(p_{n}\right) \in R S(-\alpha)$ with $\alpha>3$, then $t_{3, n} / p_{n} \rightarrow 3\left(\mu_{e} / \mu\right)^{2}$.

\section{Consecutive approximations for $\left(u_{n}\right)$}

Since $u_{m, n}=\sum_{k=0}^{m} t_{k, n}$ and $\Delta u_{m, n}=u_{m, n-1}-u_{m, n}=\sum_{k=1}^{m} \delta_{k, n}$, Lemma 2 yields the following approximations for $u_{n}$ and $\Delta u_{n}=u_{n-1}-u_{n}$ :

$$
\begin{array}{rlrl}
u_{0, n} & =\frac{1}{\mu}, & \Delta u_{0, n} & =0, \\
u_{1, n} & =u_{0, n}+\frac{1}{\mu} \bar{F}_{e, n}, & \Delta u_{1, n} & =\frac{1}{\mu} p_{e, n}, \\
u_{2, n} & =u_{1, n}-\frac{1}{\mu} R_{2, n}^{e}, & \Delta u_{2, n} & =\frac{1}{\mu} p_{e, n}-\frac{1}{\mu} r_{2, n}^{e}, \\
u_{3, n} & =u_{2, n}+\frac{1}{\mu}\left(R_{3, n}^{e}-3 R_{2, n}^{e}\right) . &
\end{array}
$$




\subsection{The approximation $\left(u_{0, n}\right)$}

Blackwell's theorem states that the renewal sequence satisfies $u_{n} \rightarrow 1 / \mu$ and $\Delta u_{n} \rightarrow 0$. In our case, (7) shows that $u_{0, n}=1 / \mu$ and $\Delta u_{0, n}=0$.

\subsection{The approximation $\left(u_{1, n}\right)$}

If $\left(\bar{F}_{n}\right) \in R S(-\alpha)$ and $\alpha>1$, then (8) and Lemma 4 show that

$$
u_{1, n}-\frac{1}{\mu}=\frac{\bar{F}_{e, n}}{\mu} \sim \frac{n \bar{F}_{n}}{\mu^{2}(\alpha-1)}
$$

and $\Delta u_{1, n}=\bar{F}_{n} / \mu^{2}$. In renewal theory, the following results are known, $\mathrm{cf}$. Theorems 3.1.6 and 3.1.7 in Frenk (1983):

- If $\left(\bar{F}_{n}\right) \in R S(-\alpha)$ with $\alpha>1$, then

$$
u_{n}-\frac{1}{\mu} \sim \frac{\bar{F}_{e, n}}{\mu} \sim \frac{n \bar{F}_{n}}{\mu^{2}(\alpha-1)} .
$$

- As in Remark 1, several authors have discussed moment conditions on $X$ or $X_{e}$ that imply $\sum_{n=1}^{\infty} n^{r}\left|u_{n}-1 / \mu\right|<\infty$ or $\sum_{n=1}^{\infty} n^{r}\left|\Delta u_{n}\right|<$ $\infty$. We refer to Feller (1949), Karlin (1955), Stone (1965), Stone and Wainger (1967), and Grübel (1979) for such type of results.

\subsection{The approximation $\left(u_{2, n}\right)$}

If $\left(p_{n}\right) \in R S(-\alpha)$ with $\alpha>3$, then (9) and Theorem 7 show that

$$
u_{2, n}-\frac{1}{\mu}-\frac{\bar{F}_{e, n}}{\mu}=-\frac{R_{2, n}^{e}}{\mu} \sim-\frac{2 \mu_{e}}{\mu^{2}(\alpha-1)} n p_{n},
$$

and

$$
\Delta u_{2, n}-\frac{p_{e, n}}{\mu}=-\frac{r_{2, n}^{e}}{\mu} \sim-\frac{2 \mu_{e}}{\mu^{2}} p_{n} .
$$

From Remark 2, it follows that $E\left(X_{e}^{r+1}\right)<\infty$ implies that

$$
\sum_{n=1}^{\infty} n^{r}\left|u_{2, n}-\frac{1}{\mu}-\frac{1}{\mu} \bar{F}_{e, n}\right|<\infty .
$$

The following results are known in renewal theory: 
- Theorem 3.1.12 of Frenk (1983) and Theorem 2.1.25 of Frenk (1987) provide conditions under which

$$
u_{n}-\frac{1}{\mu}-\frac{1}{\mu} \bar{F}_{e, n} \sim-\frac{2 \mu_{e}}{\mu^{2}} \bar{F}_{n} .
$$

See also Rogozin (1973).

- Theorem 3.1.14 of Frenk (1983) and Theorem 2.1.16 of Frenk (1987) also consider the case where $\left(p_{e, n}\right) \in R S(-\alpha)$ with $1<\alpha<2$.

- Theorem 2.1.20 in Frenk (1987) provides a sufficient condition of the type $E\left(X^{r+1}\right)<\infty$ for

$$
\sum_{n=1}^{\infty} n^{r}\left|u_{n}-\frac{1}{\mu}-\frac{1}{\mu} \bar{F}_{e, n}\right|<\infty .
$$

\subsection{The approximation $\left(u_{3, n}\right)$}

For $\left(p_{n}\right) \in R S(-\alpha)$ with $\alpha>3,(10)$ and Theorem 9 lead to the following approximation:

$$
u_{3, n}=\frac{1}{\mu}+\frac{1}{\mu} \bar{F}_{e, n}-\frac{1}{\mu} R_{2, n}^{e}+\frac{3 \mu_{e}^{2}}{\mu^{2}} p_{n}+o(1) p_{n} .
$$

We have found no similar result for the renewal sequence in the renewal literature. The approximation $u_{3, n}$ implicitly appears in Section 4 of Grübel (1983). See also Essén (1973) and Ney (1981).

\section{Concluding remarks}

1. In this paper we studied the renewal sequence when $p_{n}$ is regularly varying and thus bounded by a power of $n$. This approach does not cover exponential cases such as $p_{n}=C e^{-n}$ or $p_{n} \sim n^{\alpha} c^{n}, 0<c<1$. In a forthcoming paper we will also study this case.

2. In many cases the condition $\left(a_{n}\right) \in R S(-\alpha)$ can be replaced by the condition $\left(a_{n}\right) \in O R S \cap L S$, where ORS is the class of sequences $\left(a_{n}\right)$ for which (4) is replaced by $\forall y>0: a_{[x y]}=O\left(a_{[x]}\right)$ as $x \rightarrow \infty$.

3. To study the asymptotic behaviour of $\delta_{3, n}$ we should impose conditions on $p_{n}-p_{n-1}$. The details are somewhat complicated and further research is needed here. 


\section{References}

Bingham, N.H., Goldie, C.M., Teugels, J.L., 1987. Regular Variation. Encyclopedia of Mathematics and its Applications, Cambridge University Press.

Essén, M., 1973. Banach algebra methods in renewal theory. J. Analyse Math. $26,303-336$.

Feller, W., 1949. Fluctuation theory of recurrent events. Trans. Am. Math. Soc. $67,98-119$.

Frenk, J.B.G., 1983. On renewal theory, Banach algebras and functions of bounded increase. Ph.D. Thesis, Mathematisch Centrum Amsterdam.

Frenk, J.B.G., 1987. On Banach algebras, renewal measures and regenerative processes. Centre for Mathematics and Computer Science, CWI Tract 38, Amsterdam.

Geluk, J.L., de Haan, L., 1987. Regular variation, extensions and Tauberian theorems. Centre for Mathematics and Computer Science, CWI Tract 40, Amsterdam.

Grübel, R., 1979. Über die Geschwindigkeit der Konvergenz beim Erneuerungssatz und dem Hauptgrenzwertsatz für Markofketten. Ph.D. thesis, Üniversität Essen.

Grübel, R., 1983. Functions of discrete probability measures: rates of convergence in the renewal theorem. Z. Wahr. verw. Geb. 64, 341-357.

Karlin, S., 1955. On the renewal equation. Pacific J. Math. 5, 229-257.

Mitov, K.V., Omey, E., 2014. Intuitive approximations for the renewal function. Statistics and Probability Letters 84, 72-80.

Ney, P., 1981. A refinement of the coupling method in renewal theory. Stoch. Proc. Appl. 11, 11-26.

Rogozin, B.A., 1973. An estimate of the remainder term in limit theorems of renewal theory. Theory Prob. Appl. 18, 662-677.

Stone, C., 1965. On moment generating functions and renewal theory. Ann. of Math. Stat. 36, 1298-1301. 
Stone, C., Wainger, S., 1967. One-sided error estimates in renewal theory. Journal d' Analyse Math. 20, 325-352. 